\title{
Improvements to Image Magnification
}

\author{
A. Biancardi, L. Lombardi and V. Pacaccio \\ Università di Pavia, DIS, Via Ferrata 1, I-27100 Pavia, Italy
}

\begin{abstract}
The main limitation of current magnifying techniques is that they do not introduce any new information into the original image. This lack of information is responsible for the perceived degradation of the enlarged image. The idea underlying this work is to estimate missing frequencies from the original low resolution image and to synthesize them. Sub-pixel edge estimation and a polynomial interpolation step are the key techniques of the proposed method. Furthermore, a new extension to color images is presented. Results are encouraging even if they suggest that further effort should be spent in improving edge localization accuracy.
\end{abstract}

\section{Introduction}

Image definition is the amount of performable detail with a given resolution and it is a key factor in perceived-quality assessment. Preserving definition in image transforms is a demanding task because it sits on the overlapping between two domains: the physical and the perceptual ones.

Interpolation methods, usually employed in image magnification, cause a degradation in the enlarged image due to definition loss. In this paper we developed a new magnifying detail-preserving technique. The input of the problem is a low resolution (LR) image, with its finite information content, and the output is a high resolution (HR) version of the same image. The research goal is to produce a perceived high quality in the high resolution image, i.e. an image that should be perceived at least as nicely as the low resolution one by a human observer.

As edges are high spatial frequency features, they strongly affect the perceived image sharpness and quality. If we assume that the low resolution image is as a subsampled version of the high resolution one, we can estimate where the edges "were" before subsampling and then use this spatial information to reconstruct as exactely as possible the missing information. Therefore, edge estimation must be sub-pixel and the reconstruction of the HR image must keep into account the estimation results. The idea consists in modifying a usual intepolation scheme, e.g. bilinear or bicubic interpolation, in order to prevent interpolation across edges.

\section{Sub-Pixel Edge Estimation}

Our sub-pixel edge estimation is based on the Allelbach and Wong's work [1]. Problem analysis revealed that estimation accuracy is the most important prob- 
lem in image magnification because edge localization strongly affects the enlarged image quality. The authors proposed a linear approach which we have found to be error prone. Starting from a center-on-surround-off filtered LR image they linearly interpolate the zero-crossing $(\mathrm{ZC})$ points by a heuristic procedure which depends on the sign geometry of the low resolution pixels; then they quantize the analytical estimated edge to the high resolution grid.

Contrary to Allelbach and Wong, we found that both rotational invariance and localization of the filter strongly affect the edge-map accuracy, and hence we looked for a trade-off between these two properties, developing a $5 \times 5$ filter which we called ROT. This filter, built by discretization of a positive constant diskshaped region with a constant negative surround ring, mimics the well known LOG, which is rotationally invariant, but with more localized response due to its smaller dimension. Of course the non-directional property of the Laplacian is lost in discretization; thus also the ROT filter is not non-directional, but we found that it has an increased sensitivity along diagonals and other angles if compared to the simple rectangular filter proposed in [1].

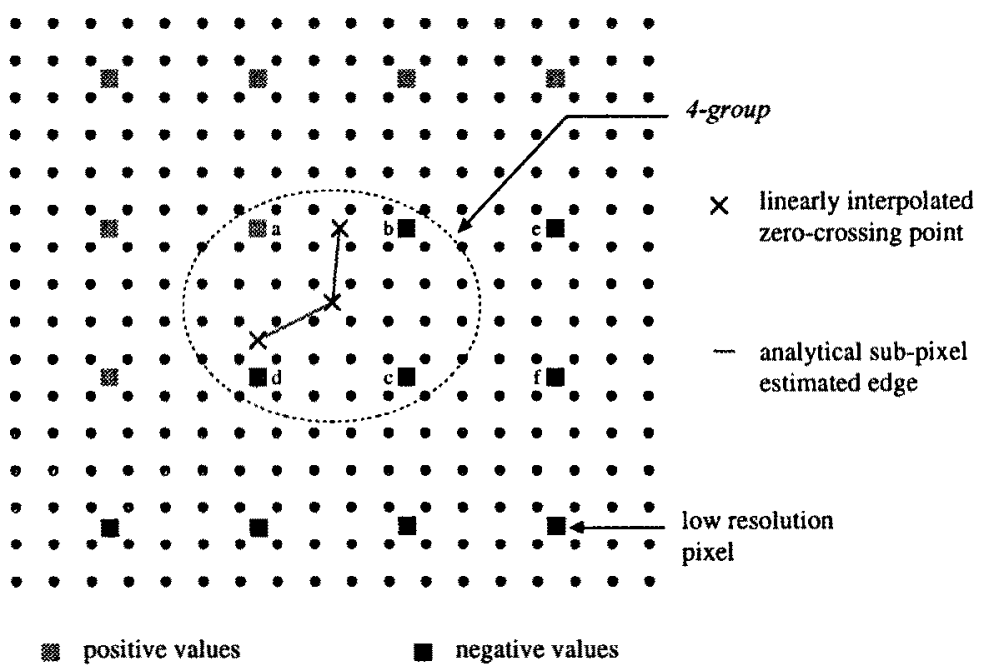

Fig. 1. Linear sub-pixel edge estimation.

Once the low resolution image has been ROT-filtered the ZCs are estimated according to the Allelbach and Wong's idea but using bicubic interpolation instead of the linear one. Referring to figure 1, the linear sub-pixel estimation procedure may be summarized as follows: for each group of $4 \mathrm{LR}$ pixels (the 4-group) of the filtered image, according to their sign geometry, sub-pixel ZCs are estimated by linear interpolation between LR pixel pairs a-b, a-c and a-d.

Unfortunately the simple linear approach is not accurate, thus we modified the estimation scheme considering the bicubically interpolated filtered image. 


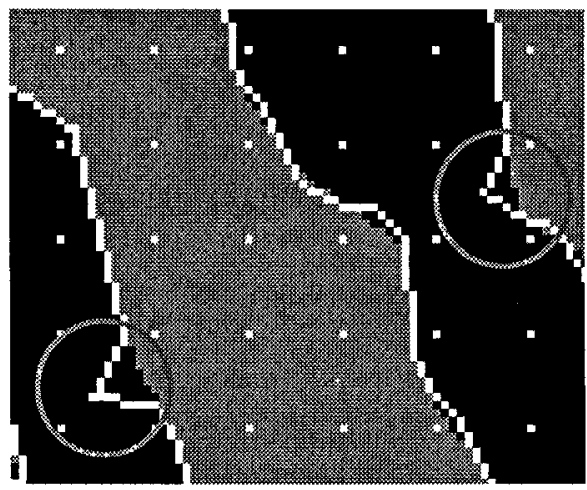

Fig. 2. Comparison between linear estimation approach and bicubically interpolated filtered image.

Edges are still represented as joint segments as well as in Allelbach and Wong's approach, but ZCs are computed by mean of bicubic interpolation which is much more accurate mainly along diagonals. Figure 2 shows the comparison between the linear estimated edge-map (white lines), and the bicubically interpolated ROT-filtered image (regions); the isolated points are the LR pixels. Circles point out that significant differences are prominent in diagonal directions, while horizontal and vertical estimates are substantially good. The figure also shows how, where linear boundary estimations conincide to the bicubic ones, the piecewise linear boundary representation appears somehow smooth, while preserving all the advatages of a representation by 1st order curves.

A great advantage of such a piecewise linear boundary description is its flexibility, which may be exploited in order to solve another important problem in edge-map estimation, i.e. the disagreement between the estimated edge positions and real region boundaries (true edges) in the LR image. Unmatching information leads to undesired resuts in interpolation. Allelbach and Wong's preprocessing step tries to solve this problem modifying the original image according to edge-map estimations. We found that this method creates artifacts in many real images. Furthermore, modifying the original image according to edge estimation results introduces the estimation error inside the original data, so that no correction will be possible. Unfortunately an edge correction scheme is not yet available due to the non-local nature it should have, so, while investigating on such a global corrector, we improved the preprocessing step: we exploited edge-strength information from first derivative edge detection in order to replace only pixels on steep edges.

\section{Rendering the Surface}

Rendering is the final step in which we reconstruct the high resolution image filling the absent information inside each 4-group by an EDI scheme. The es- 
sential feature of this strategy is that the interpolation process is modified to handle interpolation across edges, while, when no edges are found, a simple bilinear interpolation is performed. ${ }^{1}$ When there is an edge inside the current 4-group, the rendering procedure attempts to reconstruct the surface according to a slope-controllable edge model.

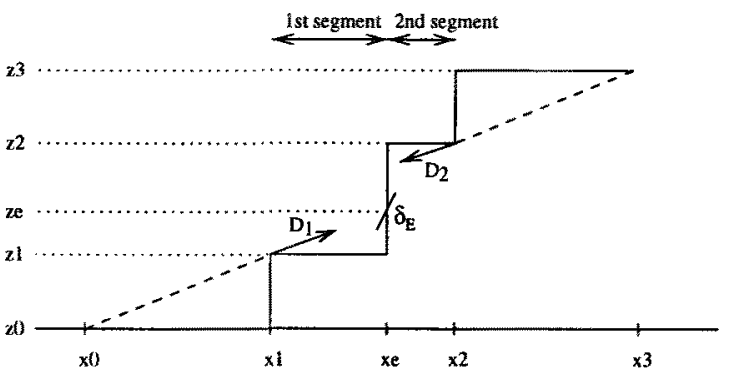

(a)

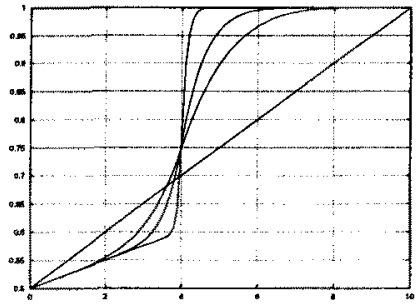

(b)

Fig. 3. (a) General 1-D situation of polynomial interpolation; (b) Example plot of the polynomial curve at different values of $n$.

The figure 3.a describes the typical 1-D situation. $x_{0}, x_{1}, x_{2}, x_{3}$ are LR pixel positions and $z_{0}, z_{1}, z_{2}, z_{3}$ are their respective gray levels. $x_{e}$ is the estimated $\mathrm{ZC}$ point. Our idea is to fit a polynomial curve that has a controllable slope $\delta_{E}$ across the edge transition, and that must interpolate the points $\left(x_{1}, z_{1}\right)$ and $\left(x_{2}, z_{2}\right)$. The point $z_{e}$ is the HR image value, i.e. the interpolating polynomial value, across the edge, and it represents a free parameter of the algorithm. In order to have some correlation between the reconstructed image in the $\left[x_{1}, x_{2}\right]$ range and neighbor LR pixels, we choose to drive the interpolating polynomial shape by its first derivatives $D_{1}$ and $D_{2}$ in the range extrema $x_{1}$ and $x_{2}$ respectively. These derivatives are computed as $D_{1}=z_{1}-z_{0}$ and $D_{2}=z_{2}-z_{3}$.

\subsection{Polynomial Interpolation}

The essential feature that the interpolating polynomial should have is the absence of oscillations whatever values the edge slope may have. Observing that the maximum slope depends on polynomial degree and oscillations depend on intermetiate degree terms, we use the following polynomial curves

$$
\begin{aligned}
& \mathcal{Z}_{1}(t)=\Delta_{1}\left[\hat{D_{1}} t+\left(1-\hat{D_{1}}\right) t^{n}\right]+z_{1}, \text { for } x \in\left[x_{1}, x_{e}\right] \\
& \mathcal{Z}_{2}(s)=\Delta_{2}\left[\hat{D}_{2} s+\left(1-\hat{D_{2}}\right) s^{n}\right]+z_{2}, \text { for } x \in\left[x_{e}, x_{2}\right]
\end{aligned}
$$

${ }^{1}$ Higher degree interpolation is not needed because no high frequency components are present (edges) so that the bilinear interpolation, which is low-pass in its nature, does not cause blurring. 
which represent the two joint curve segments in $\left[x_{1}, x_{2}\right]$, and where

$$
\begin{aligned}
& x \in\left[x_{1}, x_{e}\right] \Leftrightarrow x=x 1+t\left(x_{e}-x_{1}\right) \text { and } t \in[0,1] \\
& x \in\left[x_{e}, x_{2}\right] \Leftrightarrow x=x 2+s\left(x_{2}-x_{e}\right) \text { and } s \in[0,1]
\end{aligned}
$$

Let $\Delta_{E}=z_{2}-z_{1}, \hat{D_{1}}=D_{1} / \Delta_{1}$, and $\hat{D_{2}}=D_{2} / \Delta_{2}$; it may be easly proved that interpolation conditions are satisfied. Then we assign $C^{0}$ and $C^{1}$ continuity on the common point $\left(x_{e}, z_{e}\right)$ by fixing $\Delta_{1}=\Delta_{2}+\Delta_{E}$ for $C^{0}$ and $\mathcal{Z}_{1}^{\prime}(1)=-k \mathcal{Z}_{2}^{\prime}(1)$ for $C^{1}$, where $k=\left(x_{e}-x_{1}\right) /\left(x_{2}-x_{e}\right)$ is a normalization factor. Finally, computing first derivatives and doing substitutions, we get

$$
\begin{aligned}
& \Delta_{2}=\frac{(n-1)\left(D_{1}+k D_{2}\right)}{(1+k) n}-\frac{\Delta_{E}}{1+k} \\
& \Delta_{1}=\Delta_{2}+\Delta_{E} .
\end{aligned}
$$

It should be noted that the continuity implies that the value $z_{e}$ depends on the other parameters; this is not a problem because $z_{e}$ is a dummy point and it does not affect the perceived final image quality.

The polynomial degree $n$ must somehow be bounded to the LR edge slope in order to exactly reproduce the HR transition. Roughly speaking the higher is the edge step $\Delta_{E}$ the higher is the probability the edge to be sharp, then the higher should be $n$. Anyway, we must consider that the effect of varying the exponent depends also on the magnification factor, because the polynomial curve with a low value of $n$ affects a larger number of HR pixels, due to its flatter shape. By now we employ an heuristic relationship to bound $n$ to the edge step height $\Delta_{E}$ and to the magnification factor $x_{2}-x_{1}$, but we plan to gather and exploit some other information about the LR edge slope to improve the reproduction fidelity.

\subsection{2-D Extension}

Referring to figure 4.a and 4.b we compute the interpolating surface according to an heuristic procedure which ensures the desired slope across the edge and $C^{0}$ continuity among patches. Given the the HR pixel position, the interpolating segment through it, which is parallel to the edge, intercepts the border and the diagonal in points $p$ and $q$ respectively, hence the 1-D polynomial curve is evaluated in those points and computed values are used to linearly interpolate the HR pixel value.

\section{Mixing Edges and Smooth Areas}

A great disadvantage of $\mathrm{ZC}$ edge maps is that they do not carry any information about the edge strength, i.e. its slope. Roughly speaking the underlying assumption about the step-like shape of edges we attempt to reconstruct might be not verified where the real edge is more than one pixel wide. On the other hand we found that bicubic interpolation is suitable in such a case. Hence we compute 


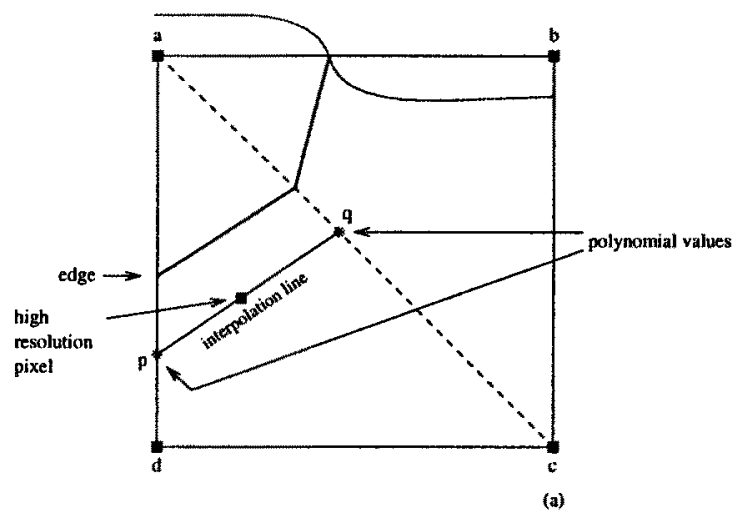

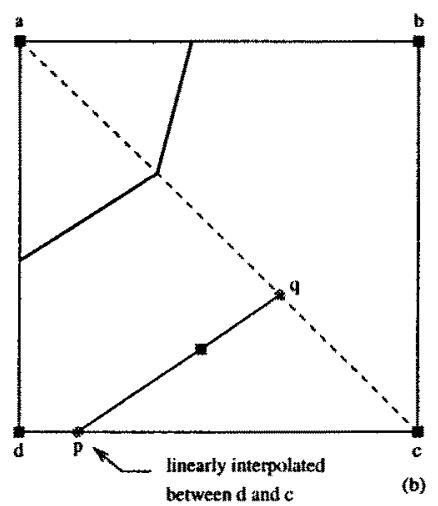

Fig. 4. 2-D extension of polynomial interpolation, example of geometry. A 4-group with its piecewise linear edge inside is shown. (a) Intercepted points (e.g. $p$ and $q$ in figure) are both on a border where a polynomial curve lies. (b) When an intercepted point is on a border where the polynomial curve does not lie (e.g. $p$ in figure), its value is linearly interpolated between LR pixel values (e.g. $c$ and $d$ in figure).

the final result, say $R(i, j)$, by linearly mixing the polynomial synthesized image, say $I(i, j)$, and the bicubically interpolated one, say $B(i, j)$. Weights, between 0 and 1 , are choosen to be a $\mathrm{HR}$ version of the first derivative $F D(i, j)$ of the LR image:

$$
R(i, j)=F D(i, j) \cdot I(i, j)+[1-F D(i, j)] \cdot B(i, j)
$$

foreach $i, j$ in the HR space.

\section{Extension to Color Images}

There is evidence that the human visual system's (HVS) color coding process is based on three visual channels, one type is independent on the wavelenght, yet the other two have a chromatic sensitivity. Moreover, there seems to be general agreement that spatial resolution is markedly lower in chromatic channels than in the achromatic one, hence high frequency informations, i.e. edges, come mainly from this channel [5-7].

Another important consideration is that, in order to avoid chromatic artifacts in the enlarged image, a non-linear operator cannot be applied to each RGB component separately. The proposed technique is strongly non-linear due to the edge detection procedure.

Hence the edge-map is built from the luminance image according to the HVS's spatial sensitivity, and directs the subsequent interpolation step on each RGB component separately. Artifacts due to color aberrations are negligible. The method can be improved by exploiting color information for a more accurate segmentation process and using perceptually uniform color spaces but which separate chrominance from luminance $[6,4]$. 


\section{Results}

We tried our algorihm with many real images and results show that image definition is well preserved, even if magnification factors are quite large, i.e. 4-8 along each dimension. Figure 5 shows a particular of a gray scale real image: the original image was magnified 8 times along each dimension, 64 times the surface, by bicubic interpolation and by our proposed method. The comparison between the two enlarged images exhibits a noticeable difference: boundaries in our image are quite sharp while a strong blurring effect is present in the bicubically interpolated one.
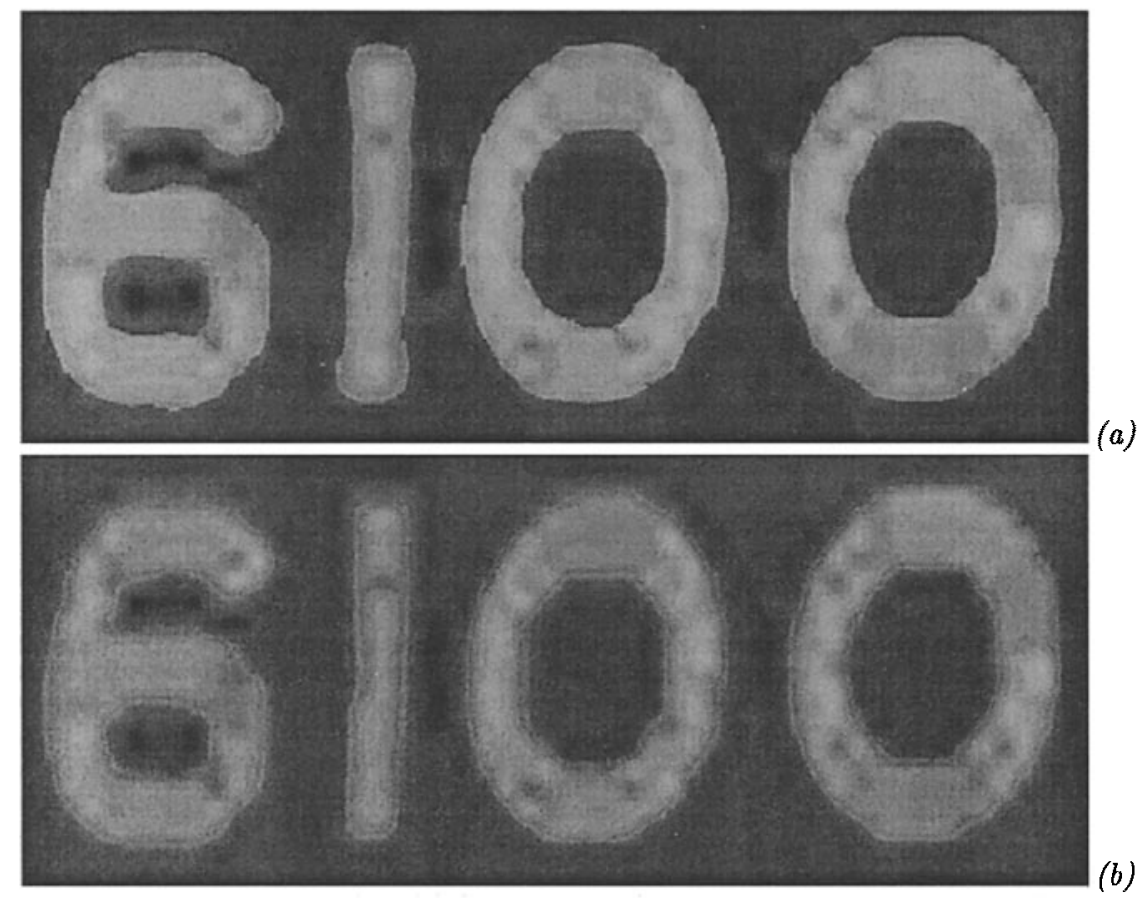

Fig. 5. (a) Proposed magnifying method; (b) Bicubic interpolation.

\section{Conclusions}

The problem of recovering the high frequency (HF) contents of a magnified image was tackled at first by reviewing existing proposals and learning about the models of human visual perception. The procedural starting point was chosen to be the work by J. Allelbach and P. W. Wong. Their methodology was studied and verified on some non-synthetic images. Main point of this methodology is 
the idea of using sub-pixel estimations of edges as the most probable locations with high HF contents. This estimation is carried out by interpolating the result of a center-on-sorround-off filter and then looking for ZCs in the filtered image.

Unfortunately the filter design is very critical because it must be as irrotational as possible and as insensitive to noise as possible. A new version of such a filter is proposed in order to minimize edge displacements caused by the space discretization of digital images. Additionally some pre-processing filters were tried with some improvements on most images.

Secondly, the comparison between a bicubically interpolated filtered image and the linear sub-pixel extimation showed that the linear approach is error prone, leading to artifacts in the magnified image.

The methodology has been extended to color images thanks to a unified (monochrome) edge map; artifacts due to color aberrations are negligible.

A great advantage of our approach is the possibility to further sharpen the magnified image with an edge-enhancing filter [8] processing that is not possible with standard (e.g. bilinear or bicubic) interpolations.

Current results are encouraging (when magnifying each image dimension by 4-8 times, i.e. 16-64 times the surface). Once the remaining sources of artifacts will be removed, this scheme will lead to a crisp and pleasing final result.

\section{Acknowledgments}

We wish to thank Dr. Lorenzo Coslovi (Hewlett-Packard Italia) for his kind support.

\section{References}

1. J. Allelbach, P.W. Wong, Edge-Directed Interpolation, Proc. ICIP-96, IEEE Press, Lausanne CH, 1996, vol. III, pp. 707-710.

2. J.D. Fahnestok, B.R. Hunt, The Maintenance of Sharpness In Magnified Digital Images, CVGIP 27, 1984, pp. 32-45.

3. R.G. Keys, Cubic Convolution Interpolation for Digital Image Processing, IEEE Trans. ASSP, vol. 29, no. 6, December 1981, pp. 1153-1160.

4. J.D. Foley, A. van Dam, S.K. Feiner, J.F. Hughes, Computer Graphics, principles and practice, second edition, Addison-Wesley, 1992.

5. A.B. Watson, Perceptual-components architecture for digital video, J. Opt. Soc. Am. A, vol. 7, no. 10, October 1990, pp. 1943-1954.

6. M. Gross, Visual Computing, Springer-Verlag, Berlin, 1994.

7. D.J. Granrath, The role of human visual models in image processing, Proc. of the IEEE, vol 69, no. 5, May 1981, pp. 552-561.

8. D.E. Knuth Digital Halftones by Dot Diffusion, ACM Transactions on Graphics, vol. 6, no. 4, October 1987, pp. 245-273. 